\title{
Paving therapeutic avenues for FOXG1 syndrome: untangling genotypes and phenotypes from a molecular perspective
}

Ipek Akol' 2,3* , Fabian Gather ${ }^{*}$, Tanja Vogel ${ }^{1,3,4}$

${ }^{1}$ Institute for Anatomy and Cell Biology, Dept. Molecular Embryology, Medical Faculty, University of Freiburg, 79104 Freiburg, Germany

${ }^{2}$ Faculty of Biology, University of Freiburg, 79104 Freiburg, Germany

${ }^{3}$ Center for Basics in NeuroModulation (NeuroModul Basics), Medical Faculty, Albert-Ludwigs-University Freiburg, 79104 Freiburg, Germany

${ }^{4}$ Corresponding author: Institute for Anatomy and Cell Biology, Dept. Molecular Embryology, Medical Faculty, University of Freiburg, Albertstr. 17, 79104 Freiburg, Germany, tanja.vogel@anat.uni-freiburg.de

*These authors contributed equally

\section{Abstract}

Development of the central nervous system (CNS) depends on accurate spatiotemporal control of signalling pathways and transcription programs. Forkhead Box G1 (FOXG1) is one of the master regulators that plays fundamental roles in forebrain development, from the timing of neurogenesis to the patterning of the cerebral cortex. Mutations in the FOXG1 gene cause a rare neurodevelopmental disorder called FOXG1 syndrome, also known as congenital form of Rett syndrome. Patients presenting with FOXG1 syndrome manifest a spectrum of phenotypes ranging from severe cognitive dysfunction and microcephaly to social withdrawal and communication deficits with varying severities. To develop and improve therapeutic interventions, there has been considerable progress towards unravelling the multi-faceted functions of FOXG1 in neurodevelopment and pathogenesis of FOXG1 syndrome. Moreover, recent advances in genome editing and stem cell technologies, as well as increased yield of information from high throughput omics opened promising and important new avenues in FOXG1 research. In this review, we provide a summary of clinical features and emerging molecular mechanisms underlying FOXG1 syndrome, and explore disease-modelling approaches in animals and human-based systems to highlight prospects of research and possible clinical interventions.

\section{Keywords:}

Rett syndrome, FOXG1 syndrome, neurodevelopmental disorders, disease modelling, hiPSCs, organoids, FOXG1, brain development 


\section{Introduction}

FOXG1 syndrome (OMIM \#613454) is a rare and severe neurodevelopmental disorder caused by heterozygous de novo mutations in the gene encoding for the transcription factor Forkhead Box G1 (FOXG1). FOXG1 has fundamental and non-redundant roles in brain development from the timing of neurogenesis to the patterning of the cerebral cortex [1], [2]. It has been previously classified as a congenital variant of Rett Syndrome (RTT, OMIM \#312750) due to clinical similarities. Nevertheless, a combination of developmental and anatomical features distinguish FOXG1 syndrome from the typical Rett syndrome, which is caused by mutations in the X-linked gene encoding for the transcriptional repressor methyl-CpG-binding protein (MECP2) [3]. Compared to typical Rett syndrome, the FOXG1 syndrome shows earlier onset in patients with a complex spectrum of phenotypes comprising microcephaly, corpus callosum agenesis, delayed myelination, seizures, disrupted circadian rhythm, social withdrawal and severe intellectual disability with poor or absent speech development [4], [5]. Additionally, FOXG1 syndrome is associated with autism spectrum disorders (ASD) and FOXG1 variants are identified in patients with ASD [6]. However, due to its variable and broad spectrum of phenotypes FOXG1 syndrome remains underdiagnosed, which consequently limits the research on its aetiology and potential therapeutic interventions.

As of October 2021, 860 cases of FOXG1 syndrome are reported worldwide, with the number of diagnosed individuals increasing as genetic testing becomes more prevalent [7]. Thus far, the identified mutations associated with FOXG1 syndrome include chromosomal microaberrations such as deletions and duplications, as well as frameshift and point mutations [4], [5], [8]-[10]. Depending on the type of mutation, a variability in phenotypic manifestations has been observed [4], [5], [11], [12]. The most severe phenotypes occur in patients with frameshift or nonsense mutations in the $\mathrm{N}$-terminal domain, including the Forkhead domain, while milder phenotypes associate with FOXG1 missense mutations in the Forkhead domain [5]. As this genetic variability in patients makes it difficult to pinpoint the direct and indirect outcomes of identified mutations in the FOXG1 gene, the challenge remains to dissect genotype-phenotype associations. Functional variability of residual FOXG1 and contribution of dosage-effects to 
the syndrome make it even more difficult to disentangle the aetiology of the syndrome and the diverse molecular functions of FOXG1. In this review, we discuss the clinical features and possible correlation to the seemingly diverse molecular alterations underlying FOXG1 syndrome, and explore up-to-date disease models aiming to advance potential therapeutic avenues.

\section{Clinical manifestations of FOXG1 syndrome}

The first heterozygous de novo translocation mutation in FOXG1 was identified in a 7-yearold patient in 2005 [13]. The patient manifested microcephaly, complete agenesis of the corpus callosum, and cognitive disability. Shortly after, two other individuals fulfilling criteria for Rett syndrome variants were diagnosed with carrying mutations in the FOXG1 gene [14]. Because of a substantial phenotypic overlap between the condition caused by mutations in the FOXG1 gene and typical Rett syndrome, mutations in FOXG1 were classified as a congenital variant of Rett syndrome [14], [15]. However, increasing numbers of patients diagnosed with FOXG1 mutations nowadays allowed refinement of the phenotypic manifestations and classification of this condition as FOXG1 syndrome. Among the phenotypes of FOXG1 syndrome that diverge from typical Rett syndrome were the earlier (congenital) onset, specific brain imaging abnormalities, dyskinesia and lack of regression, which comprised the hallmarks used to distinguish FOXG1 syndrome from typical Rett syndrome [4], [5], [11]. Following studies and several clinical screens laid further foundation to specify the FOXG1 syndrome, which is now recognised as a distinct human neurodevelopmental disorder.

Despite significant variability in phenotypes and severity observed in individuals depending on the genotype, the core FOXG1 syndrome phenotype consists of severe postnatal microcephaly and mental retardation, deficient language development, poor social interactions and eye contact (indicating a link to ASD), postnatal growth deficiency, problematic sleep patterns, epilepsy, and irritability during infancy [4], [12]. Additionally, data from brain imaging studies uncovered corpus callosum agenesis and reduced white matter, as well as poor and delayed myelination patterns [4], [5], [11]. 
In humans, FOXG1 is located on chromosome $14 q 12$ and contains only one coding exon [16], [17]. Among vertebrates, the $\mathrm{N}$-terminal domain does not display a large degree of evolutionary conservation [18]. In contrast, the amino acid sequence from the Forkhead domain (FKHD) to C-terminal domain is highly conserved [16], [19]. The FKHD mediates binding of FOXG1 to the DNA. In addition to the FKHD, the FOXG1 protein harbours a 20residue Groucho (Gro)-binding domain (GBD) and a 10-residue histone demethylase (KDM5B/JARID1B)-binding domain (JBD) within the C-terminal part (Figure 1). A striking feature of the $\mathrm{N}$-terminal domain is its contribution to DNA-binding in addition to the classical FKHD. Thereby, FOXG1 seems to recognise and bind to canonical, FKHD recognition binding motifs, and to alternative, non-canonical DNA sequences [20]-[22]. Notably, other members of the FOX transcription factor (TF) family also bind or recognise DNA through both their FKHD and the variable $\mathrm{N}$-terminal protein domain, which indicates generally important roles of other domains of the protein than the FKHD. However, these roles are not fully understood as of yet.

Since the first identified case of FOXG1 syndrome [13], more patients with mutations in FOXG1 were diagnosed utilising chromosomal microarray, whole exome sequencing (WES), and gene sequencing methods [1], [4], [5], [7], [11], [12]. As mentioned before, FOXG1 mutations encompass missense, nonsense, and frameshift mutations as well as microdeletions proximal to or spanning the FOXG1 gene [4], [5], [29], [30], [36]-[38]. Although the mutations distributed throughout the entire gene and could thus localise principally to all known protein domains, a few hotspots were eminently more susceptible to de novo mutations, as has been reported by genotype-phenotype studies [1], [4], [5], [11], [12] (Figure 2). Two of these hotspots located in stretches of cytosine and guanine repeats within the 5'-end of the gene that encodes the $\mathrm{N}$-terminal protein domain, c.256dupC and c.460dupG, respectively [4], [5], [11], [24] (Figure 2). Mechanistically, one assumes a particular susceptibility towards replication errors in this genomic sequence [25]. In addition to mutations that occurred directly in the FOXG1 gene, some patients carried micro-deletions in genomic regions that regulate FOXG1 expression. These deletions localised both up- and downstream of the coding 
sequence and led to misregulation, and consequently mostly insufficient expression of FOXG1 [8], [9], [26].

As screenings for FOXG1 syndrome became more prevalent, increasing number of variants and corresponding phenotypes have been identified, and this allowed the exploration of genotype-phenotype correlations in more detail. Several studies based on comparably large patient cohorts provided overlapping conclusions in regard to the phenotypic manifestation of FOXG1 syndrome. However, also conflicting results regarding more uncommon phenotypes were reported [4], [5], [11], [27]. Most importantly, mutations in the 5'-end and the FKHD seemingly caused more severe phenotypes compared to mutations in other localisations. Notably, the most severe outcomes were observed in patients who carried mutations bearing truncated FOXG1 protein variants [5]. On the other hand, mutations located in the 3 '-end of the gene and thus affecting the protein's C-terminus associated with milder phenotypes compared to the 5 '-end. The high degree of evolutionary conservation of the FKHD among different species already hinted towards its critical role for FOXG1 functions. It is thus expected that mutations affecting the DNA-binding domain of FOXG1 or the DNA-binding regulatory region within the $\mathrm{N}$-terminal protein domain are poorly tolerated and lead to severely dysfunctional FOXG1 variants [5], [21], [22]. Puzzling though was the finding that the mutation hotspots of c.256dupC and c.460dupG associated with great variability of features and severities. This poor correlation between genotype and phenotype might therefore indicate that further components should be considered as potentially critical modulators of the clinical phenotypes in FOXG1 syndrome. Putative, as thus far mainly unexplored, components might include the genetic background, variable epigenetic landscapes, or environmental influences [5].

It has become clear that also pleiotropic and non-redundant functions of FOXG1 are involved in a severe pathophysiology with complex genotype-phenotype relationships upon mutations [4], [5], [12]. Thus, recognition of FOXG1 syndrome as a distinct entity was of great importance for focusing upcoming research efforts, disease modelling approaches, and subsequent potential therapeutic undertakings. Further activities should be based on a clear understanding 
of the diverse functions of FOXG1, especially in human model systems, to eventually embark on therapeutic avenues to cure or facilitate living with FOXG1 mutations.

\section{Recapitulation of FOXG1 functions in brain development and function}

FOXG1, previously also called brain factor-1 (BF-1), is a winged-helix TF of the Forkhead (FKH) family. FOXG1 is uniquely expressed in the nervous system, and is active in the early telencephalon, the cerebral cortex and hippocampus, in addition to the inner ear, retina and olfactory epithelium [2], [18], [28]. It has diverse and non-redundant functions, comprising cell proliferation and progenitor pool expansion [29], regional patterning of the forebrain [30], cell migration during corticogenesis [31] and circuit assembly [29], [32]-[35] (Figure 1). Over the years, numerous studies, using conventional and conditional knockout mouse models and genome editing techniques, have established FOXG1 as a master regulator of brain development, as reviewed in detail elsewhere [1], [18]. Here, we focus on the functions and molecular aspects of FOXG1 relating to the core phenotypes of FOXG1 syndrome.

One of the best studied model systems to understand FOXG1 functions are transgenic mice. Several Foxg1 knockout mouse models were created by replacing the coding region of Foxg 1 with lacZ, cre, or tet (tetracycline transactivator) [27], [28], [35]. These models all showed severe reduction in size of the cerebral cortex and mortality at birth [28], while the haploinsufficient Foxg $1^{\mathrm{Cre} /+}$ mice survived postnatally, and exhibited microcephaly and impaired neurogenesis phenotypes in the cortex and hippocampus [37]-[39]. Therefore, the Foxg $1^{\text {Cre/+ }}$ mice served as an appropriate model to study the human FOXG1 syndrome.

The mechanisms underlying the observed hypoplasia was investigated rather extensively. Cortical stem cells featuring constitutive loss of FOXG1 exhibited a premature lengthening of the cell cycle, concomitant with an increased exit from the cell cycle, which led to neuronal differentiation [28], [29]. Both DNA-binding dependent and independent mechanisms regulated the functions of FOXG1 in cell cycle control. While the cell cycle length was dependent on DNA-binding role of FOXG1, the normal cell cycle exit required that FOXG1 antagonised the anti-proliferative activity of TGF $\beta$ by associating with DNA-binding proteins 
which function as SMAD partners [29], [40]. A decreased level of FOXG1 in intermediate progenitor cells (IPCs) was also associated with an increased expression of the cell-cycle inhibitor Cdkn1a (p21), contributing to the early exit from cell cycle [39]. Additionally, it was reported that FOXG1 antagonized FOXO/SMAD-dependent neuronal differentiation of cortical progenitors through direct association with the FOXO/SMAD complex, or by competitively binding the consensus FKH binding site [41], [42]. FOXG1 thus reduces the expression of Cdkn1a, prevents cell cycle exit and enables the continued proliferation of FOXG1 expressing cells, consequently enabling prolonged progenitor pool expansion [29], [41], [42]. Cells lacking FOXG1 differentiate into neurons prematurely, thus depleting the progenitor pool and leading to a reduction in the brain size. Together, the observed microcephaly in these mouse models was seemingly multi-faceted and involved both DNA-dependent and -independent mechanisms that subsequently comprised the regulation of cell cycle proteins and other factors.

As FOXG1 affected the early phase of corticogenesis, it was not surprising that layering defects were observed in Foxg1 knockout mice. The mammalian cerebral cortex consists of six layers of neurons that are generated in an inside-out manner, except for the first-born neurons residing in layer 1, called Cajal Retzius cells (CRC) [43], reviewed in detail elsewhere [44], [45]. In depth studies on the role of FOXG1 in corticogenesis and layering of the cortex used conditional knockouts of the murine Foxg1. Early during corticogenesis, the absence of FOXG1 caused an excessive production of CRC, and a failure to produce later-born neurons [29], [32]. Moreover, deep-layer progenitors reverted to the production of the early born CRC [32]. Under normal conditions, FOXG1 coordinated the production of different types of projection neurons through direct inhibition of the early progenitor transcriptional factor network of Tbr1, Dmrta1, Ebf2, and Ebf3, as shown by transcriptome and ChIP-seq studies [30], [32], [33]. Moreover, the expression of FOXG1 in the intermediate zone in the later stages of corticogenesis was needed for the separation of later-born subtypes of cortical neurons [34]. Thus, FOXG1 has crucial roles in mammalian cortical expansion, not only in progenitor pool expansion but also in proper layering and patterning of the cortex and subtype identities, consequently affecting the functionality of the cortex. 
Agenesis of corpus callosum is another hallmark of FOXG1 syndrome relating to cortical development, seen in varying severities in patients [4], [13]. This malformation was also observed in some Foxg1 haploinsufficient mice, while severe hypogenesis was a feature in Foxg1 knockout mice [28], [29], [35], [38]. Callosal projections connect both cerebral hemispheres and confer associative connections, disturbance of which has been also observed for example in ASD. Neurons residing in all cortical layers contribute to callosal projections [46]. Although Foxg1 expression becomes variable in post-mitotic neurons, recent studies demonstrated that heterozygous deletion of Foxg1 in mature cortical projection neurons resulted in defects in upper-layer projection neurons, concomitant to aberrant axonal projections through the corpus callosum [34], [35]. Mechanistically, FOXG1 repressed NR2F1 (COUP-TFI) expression, which transformed local projection neurons to callosal projection neurons [34]. Additionally, FOXG1 formed a repressive complex with ZNF238 (RP58, ZBTB18), and ChIP-seq analyses revealed Robo1, Slit3, and Reelin as target genes of this repressor complex, all of which are key regulators of callosal axon guidance. Thus, FOXG1 plays a crucial role in establishing callosal projections and promotes the radial migration of cortical neurons [35]. These studies provided critical insight into the molecular mechanisms behind the agenesis of the corpus callosum and identified FOXG1 as an important factor favouring cortico-cortico projections.

Over $80 \%$ of FOXG1 syndrome patients presenting with deletions or intragenic mutations of FOXG1 are diagnosed with epilepsy, rendering this feature as one core phenotype [24]. Notably, patients with FOXG1 duplications also developed epilepsy albeit to a lesser extent [24]. Thus, deciphering varying characteristics of epilepsy is important to distinguish genotypephenotype associations in patients, and to provide effective therapies. Epileptic phenotypes and seizures have also been observed in Foxg $1^{\mathrm{Cre} /+}$ mice. In vivo electrophysiological characterisation of this animal model revealed that $F o x g 1^{\mathrm{Cre} /+}$ mice showed hippocampal hyperexcitability and that they were susceptible to seizures, which was linked to decreased expression of the chloride transporter KCC2 and GABA transporter vGAT [47]. Similar insights were obtained for the Foxg1 haploinsufficient cortex, whereby decreased levels of FOXG1 led to higher excitability and depressed synaptic transmission, due to increased expression of 
vGLUT2, accompanied by decreased levels of KCC2, decreased levels of GLUA1 and PSD95, respectively [48]. Foxg $1^{\text {Cre/t }}$ mice had susceptibility to seizures both in the cortex and the hippocampus that can be linked to excitation/inhibition imbalance, while underlying mechanisms remain to be unraveled.

Another way of action for FOXG1 impinged on mitochondrial function to regulate bioenergetics. This finding implicated that FOXG1 is crucial for mitochondrial functions during embryonic development and in pathological conditions [49], and further signified that it functions beyond chromatin-mediated transcriptional regulation. Interestingly, a triheptanoinbased anaplerotic diet, which has been used previously to treat some inherited metabolic disorders including typical RTT [47], [50]-[52], rescued the altered expression of KCC2 and vGAT and normalised enhanced susceptibility to seizures [47]. Although we are still far from fully understanding the underlying mechanisms or the role FOXG1 in neuronal metabolism and whether this translates to misbalanced excitation and inhibition, these findings present a promising therapeutic approach to alleviate the epileptic symptoms of FOXG1 syndrome patients.

Along those lines, in haploinsufficient FOXG1 mice, GLUD1 (orphan glutamate receptor $\delta-1$ subunit) expression decreased alongside other GABAergic and glutamatergic markers, indicating a shift in excitation/inhibition balance [53]. Using cellular and animal models, this comprehensive study also reported a temporal shift towards a general decrease of brain synapses, although the regulatory link between FOXG1 and GLUD1 remained to be disentangled [53]. Additionally, FOXG1 associated with the microprocessor complex through its interaction with DDX5 and played a role in miRNA biogenesis of miR-200 family regulating post-transcriptionally PRKAR2B expression. PRKAR2B inhibits postsynaptic functions by interfering with PKA activity [54], implying that deregulation of PRKAR2B in FOXG1 syndrome could contribute to synaptic dysfunctions observed in patients.

Together, the function of FOXG1 at different developmental stages emphasises its importance in the proper development and function of the CNS and establishes it as an important player 
in human neurodevelopmental disorders, while its diverse mechanisms remain to be fully explored.

\section{Human-based models of FOXG1 syndrome and function}

While the constitutive knockout of FOXG1 caused prenatal death in mice [28] $]_{2}$ its haploinsufficiency only exhibited both mild microcephaly and behavioural abnormalities [32]. However, humans with heterozygous loss of FOXG1 develop variable symptoms with differing severities [4], [5], [11], [12]. Nevertheless, direct and deeper analyses of the effects of FOXG1 mutations in humans is limited ethically to medical imaging, clinical observations and postmortem analyses. Therefore, developing appropriate models for projected investigations is crucial. Investigations during the last decades opened the door to additional opportunities of modelling, especially owing to the outstanding progress in stem cell biology. Human induced pluripotent stem cells (hiPSCs) harbour a high potential for the study of neurodevelopmental diseases. Their use in basic research aims to decipher molecular alterations underlying for example CNS diseases. This model system shall provide an extended picture of potential mechanisms triggered by gene defects in human cells; allowing for experimental attempts that bypass interspecies variations. Additionally, hiPSCs offer patient-specific modelling and therapeutic adjustments for many different diseases, as they are directly generated from patient-derived fibroblasts or other cells. During the last years, research on Rett syndrome, in the context of understanding the neurodevelopmental basis, used hiPSCs and hiPSC-derived NSCs as well as neurons to analyse and describe changes in gene expression, cell activity and cell composition [55]. The differentiation of RTT-patient-derived hiPSCs into neurons led to fewer synapses, reduced spine density, and smaller soma size by reduced MECP2 expression [56]. Moreover, comparative studies focussing on typical and atypical Rett syndrome have been fuelled by hiPSC-derived technology. Mechanistic comparison between Rett syndrome caused by either MECP2 or CDKL5 mutation revealed common targets including Glutamate Dehydrogenase 1 (GLUD1), which is encoded by the GRID1 gene. Increased expression levels of GLUD1 were observed in NSCs differentiated from one patient cell line with MECP2 mutation and two cell lines with CDKL5 mutations [57]. 
In contrast to typical Rett syndrome, only few studies report so far on FOXG1 syndrome patient-derived hiPSCs. In consequence, we are missing a rich data resource to further discern mechanistically between typical Rett, atypical Rett and FOXG1 syndrome. Despite this general shortage of data reporting on molecular alterations in FOXG1 syndrome, first studies are available, in which transcriptional alterations in hiPSC-derived (from two female FOXG1 ${ }^{+/-}$ patients) NSCs were reported. The authors observed an imbalanced expression of proteins that confer excitation and inhibition in patient-hiPSC-derived NSCs. This observation fostered the conclusion that FOXG1 is an important modulator of the ratio of excitatory and inhibitory neurons [53], similar to the outcomes from the mouse model described above [47]. Therefore, it seems highly likely that misbalanced neuronal activity in regard to excitation or inhibition is one direct link to the patient's microcephalic and epileptic features. Interestingly, the same study showed also an increase of GLUD1 expression, similar to the observation of transcriptional alterations upon MECP2 and CDKL5 mutation, which hints towards commonalities between typical and atypical Rett and FOXG1 syndromes. Such overlaps might be particularly important for the rare diseases we are describing here, as they might share future therapeutic attempts [53]. However, GLUD1 was found decreased in mouse models. These contradicting findings between different model systems emphasises the power and need of using novel technology such as hiPSCs to study FOXG1 syndrome in the best model of the patients' conditions.

Thus, another promising avenue of using FOXG1 patient-derived hiPSCs and their cellular progeny is to test rescue strategies for the FOXG1 mutation. Recently, the modification of patient cell lines using an adeno-associated virus (AAV)-coupled CRISPR/Cas9 system was reported [58]. This study showed that using CRISPR/Cas9-mediated genome editing was not only effective in repairing the mutation in primary fibroblasts of two FOXG1 syndrome patients, but also in correcting the pathogenic variant in hiPSCs. Effectivity on the molecular level was indicated, for example, by normalised levels of PAX6 expression in developing neurons. With this study the authors laid the foundation for a novel approach towards CRISPR-based personalised therapy of a severe neurodevelopmental disease [58]. 
Despite the low outcome of hiPSC-driven study of FOXG1 syndrome, several hiPSCs that originate from FOXG1 syndrome patients are available for further research and they are deposited in different biobanks [59]., e.g. the Coriell Institute for Medical Research, USA or the Biobank of the University of Siena, Italy [60]. Current progress in stem cell research, especially regarding the generation of diverse types of brain organoids, gives additional opportunities to generate cellular model systems, in which, for example, dynamic spatiotemporal processes of early brain development can be mimicked. This renders stem cell research attempts very useful, to model particularly human brain disorders [61]. Cerebral organoids can give important insights into neurodevelopmental diseases affecting the forebrain [62], and could thus serve as a suitable model and research platform to advance the understanding of FOXG1 syndrome both mechanistically and clinically. As one of the first experiments in this direction, hiPSC lines and organoids from ASD patients led to the observation that increased levels of FOXG1 correlated with this disease [6]. Accordingly, treatment of these ASD mimicking organoids with different FOXG1 shRNAs rescued the observed molecular changes. Levelling the FOXG1 expression towards control conditions, restored differences in GABAergic neuronal differentiation that was observed in patientcompared to healthy donor-derived organoids. But this rescue strategy did not majorly affect the expression of dorsal forebrain marker genes or transcription factors responsible for cortical excitatory neuron differentiation [6]. Together, this ground-breaking study revealed the important role of FOXG1 in controlling the balance of neuronal subtypes in functional neuronal networks [6].

Another line of experiments was based on CRISPR/Cas9 and small molecule-assisted shutoff (SMASh) technology to modulate FOXG1 expression in hiPSCs and hiPSC-derived cells. This experimental paradigm allowed the investigation of FOXG1 function in a dosagedependent setting in which the differentiation of hiPSCs into neurons was studied, as well as the cross talk of cortical and medial ganglionic eminence organoids [63]. In accordance with the finding upon increased expression, the reduced expression of FOXG1 bore fewer inhibitory GABAergic neurons, alongside impaired maturation of this interneuron class and overall smaller brain organoid formation. The severity of the phenotypic alterations upon impaired 
FOXG1 expression correlated directly with the remaining dose of FOXG1 in the cells, emphasising that a well-balanced availability of the FOXG1 protein is critical for proper brain development and function [63]. But still, whereas Rett syndrome patient-derived hiPSCs were differentiated into organoids [64],[65], this experimental approach is thus far not reported for hiPSCs of FOXG1 patients. Overall, there are now opportunities for investigating FOXG1 syndrome in hiPSCs and different types of organoids, e.g. modelling the ventral or the dorsal telencephalon and to verify findings from mouse or other animal models. However, more importantly, these novel technologies allow getting more insights into human- and mutationspecific mechanisms driving the FOXG1 syndrome phenotypes.

\section{Future of FOXG1 syndrome modelling, therapeutic prospects and limitations}

Patient-derived hiPSCs as well as brain organoids generated from such resources are currently a very promising approach for modelling neurodevelopmental diseases including FOXG1 syndrome. While hiPSC 2D cultivation and differentiation serve as a model for the development of single cell lineages and to decipher cell lineage-dependent molecular mechanisms, 3D brain organoids add on to this, as they are a more systematic model that integrates the single cells into their quite natural network consisting of a heterogeneous variety of cell lineages [66].

The hiPSCs technology now allows studying individual mutations of FOXG1 syndrome patients in order to determine genotype-phenotype specific correlations, cellular processes and molecular mechanisms. Additionally, the advantage of CRISPR/Cas9 editing is the ability to introduce specific mutations into an even more controlled model system due to isogenic backgrounds. Therefore, studies on genome-edited hiPSCs might avoid batch effects caused by the patient specific genomic or epigenetic background. Moreover, approaches resembling those of the AAV-mediated repair of FOXG1 mutations are not only serving the generation of cell lines with the same genetic background, but also lay a foundation for individualised medicine and early gene-therapy of FOXG1 syndrome [58] (Figure 3).

Albeit promising, disease-modelling systems also have their limitations. Mice and other animals have the advantages that an entire organism and entire developmental processes 
can be studied, but they lack the human background, have limitations to mimic all facets of human CNS development, and cannot reflect human-specific gene regulation and molecular mechanism. Aspects like the basic understanding of brain development in FOXG1 syndrome may be sufficiently mirrored, but systematic outcomes and whole organism effects can just be conjectured. The same limitations, however, also apply to hiPSC-derived organoids. Nevertheless, ongoing and future development of highly structured organoids, combining various CNS regions and containing blood-vessel structures, will give rise to even better model systems. Such advanced or second-generation organoids are closer to the natural development compared to the possibilities that are the current state-of-the-art. One can hope that in the future, organoids can grow further to mimic late human development and function, to include a higher variety of neural subclasses, and thus a more complex brain/organoid structure. The first generation of vascularised organoids [67], [68] gives rise to further development, standardisation, and generation of models reflecting later neurodevelopmental steps. The generation of vascularised organoids with a potentially functional blood brain barrier [69] as well as the screening and analysis of potential drugs with blood-brain-barrier organoids [70] are promising and necessary steps towards therapeutic approaches. Moreover, fusion of brain organoids is currently used to model interactions between different brain regions [71]. The development of additional tissues, like bilateral optical vesicles [72], or even the fusion with muscle cells for the development of motoneurons and of neuromuscular junctions, [73], [74] have been performed and these studies exemplified the advantages ahead using organoids to study human brain diseases. Thus, these approaches will prove useful in the future to investigate in further detail, how FOXG1 influences later neural development, its potential influence on tissue interaction, on entire organ function, and even multi-organ interplay. The use of such "higher developmental organoids" will serve to decipher the fundamental functions of FOXG1 in neural development. In addition, they can be exploited as standardised model systems for screening potential drugs targeting symptomatic and developmental features of FOXG1 syndrome patients. Examples of similar approaches have been reported for Glioblastoma patients [75] amongst others [76], [77]. In this light, 
personalised medicine for individual FOXG1 patients could be implemented in future on the grounds of screening patient-derived organoids with common or new drugs.

Nevertheless, researchers should also be aware of ethical issues that are associated with the generation of organoids with increasing complexity and developmental structures [78].

\section{Conclusions}

FOXG1 syndrome is a severe neurodevelopmental disorder that has been studied for many years as a variant of Rett syndrome, and must therefore be considered to be underdiagnosed as an individual disease. The diversity of FOXG1 mutations, the plethora of FOXG1associated mechanisms of transcriptional and posttranscriptional regulations, and the diverse and non-redundant functions of FOXG1 underline the importance of further research into disease-relevant alterations. Research focusing on exploration of varying FOXG1-dependent molecular mechanisms on one hand, and understanding genotype-phenotype correlations on the other hand, will lead to further discrimination of FOXG1 and other Rett-like syndromes. By now, many different studies pointed out the importance of FOXG1 in spatiotemporal control of neurodevelopment, the development and composition of different neural lineages within the CNS, and resulting effects on neural plasticity. These results can be directly connected to the severe phenotypes that are observed in FOXG1 syndrome patients. The current development in stem cell biology and disease modelling offers a variety of opportunities to investigate FOXG1 function and the individual genotype-phenotype relations even further. With the help of patient-derived hiPSCs and organoids, the development of new and personalised therapeutic approaches for the FOXG1 syndrome can be improved and expedited. After an upcoming experimental phase aiming to corroborate findings of animal models in human organoids harbouring various complexity, novel technology shall embark to integrate the most important findings, and finally to pinpoint key players that one can exploit therapeutically to improve conditions to live with FOXG1 mutation. 


\section{Figures}

(i)

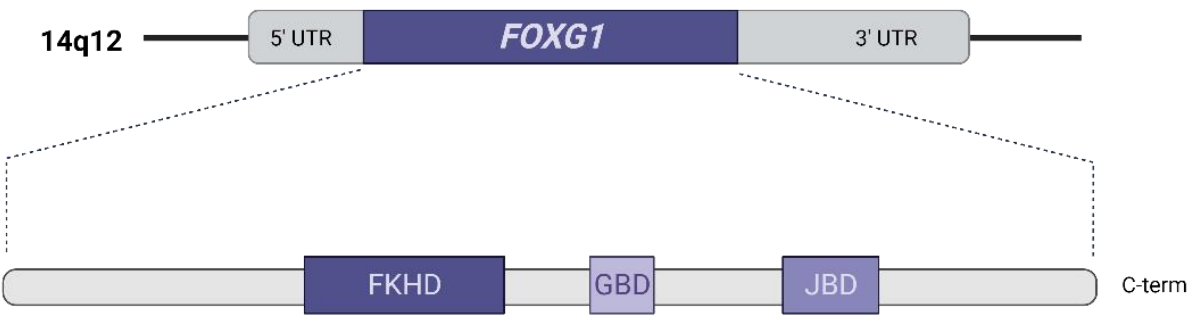

(ii) $\mathrm{N}$-term
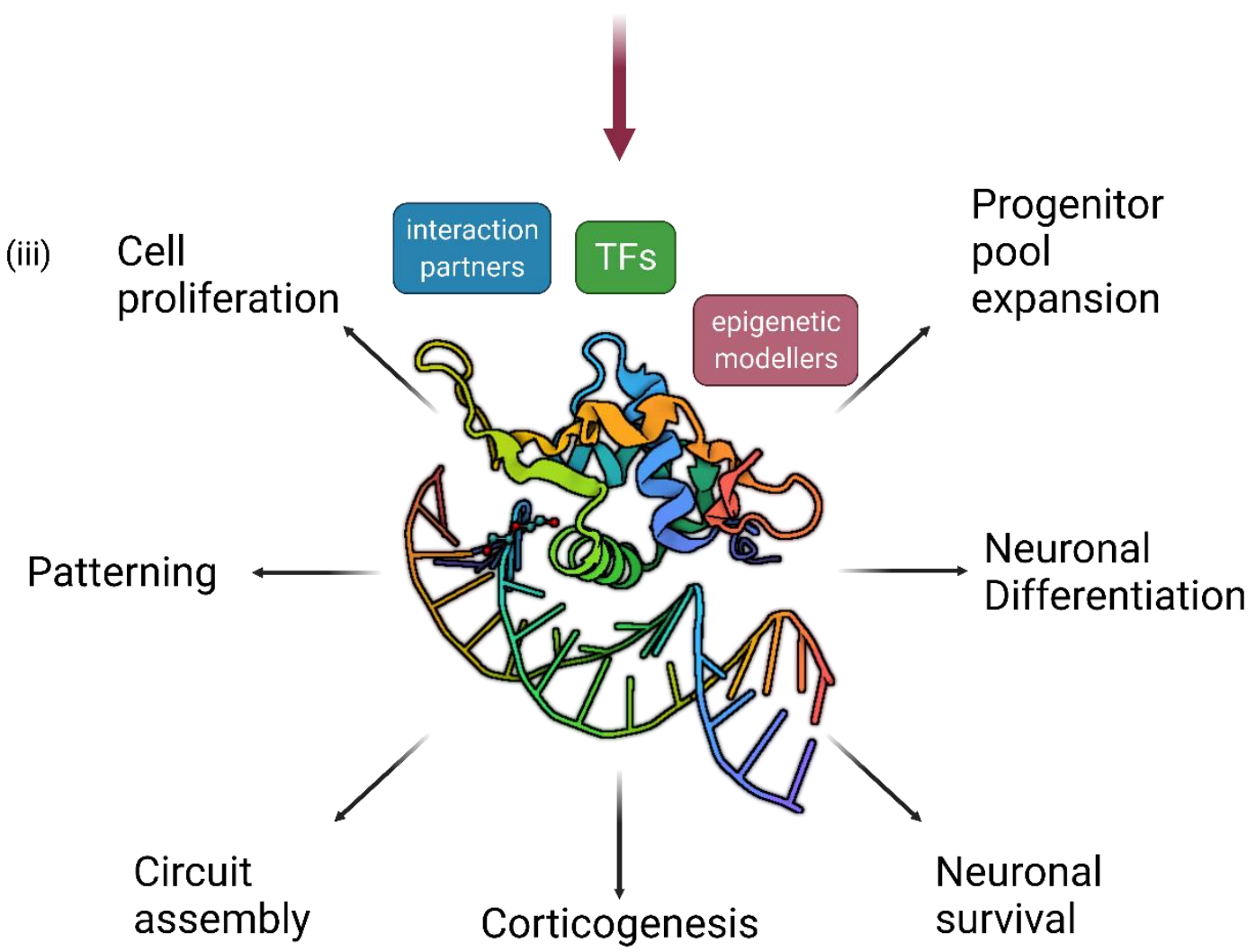

Figure 1: Functions of FOXG1. FOXG1 is located in 14q12 in humans and contains only one exon (i). FOXG1 protein domains: FOXG1 consists of a Forkhead domain (FKHD), a 20-residue Groucho (Gro)-binding domain (GBD) and a 10residue histone demethylase (KDM5B)-binding domain (JBD) (ii). FOXG1 plays important roles in many neurodevelopmental processes by its interaction with DNA and protein interaction partners (iii). Illustration was created with Biorender.com. 
(i)

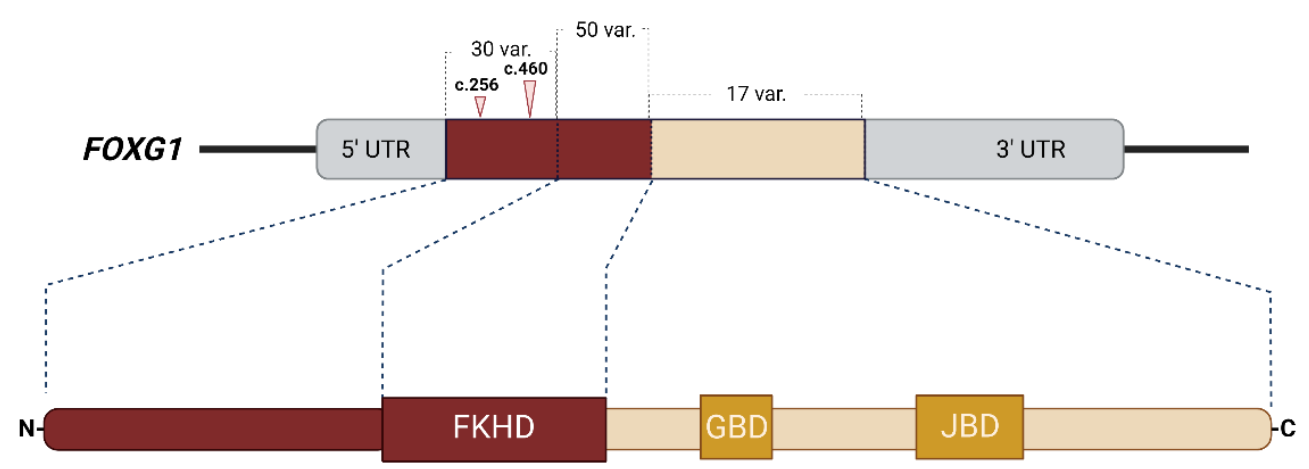

(iii) Frameshift-variants arising from hotspot mutations:

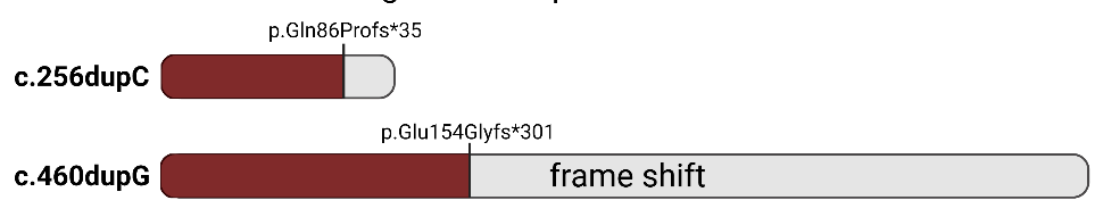

Figure 2: Mutation hotspots of FOXG1. FOXG1 gene (i) and protein (ii) domains, and the distribution of variants in a schematic illustration depicting the N-terminal domain, Forkhead binding domain (FKHD), Groucho-binding domain (GBD), JARID1B-binding domain (JBD), and C-terminal domain. The mutations are distributed in all parts of the gene, affecting all protein domains. The most severe phenotypes are observed upon mutations in the $\mathrm{N}$-terminal domain and FBD (shown in red) There are two mutation hotspots in the N-terminal region (arrows) that lead to frame shifts starting upstream of the FBD, GBD, and JBD. Potential variants arising from the mutations in the two hotspots, c.256 and c.460, are shown in (iii). The variants in the C-terminal domain, including GBD and JBD cause milder phenotypes of FOXG1 syndrome (shown in yellow). Number of variants observed in each protein domain are noted on the illustration [4], [5], [11], [12]. Illustration was created with Biorender.com. 


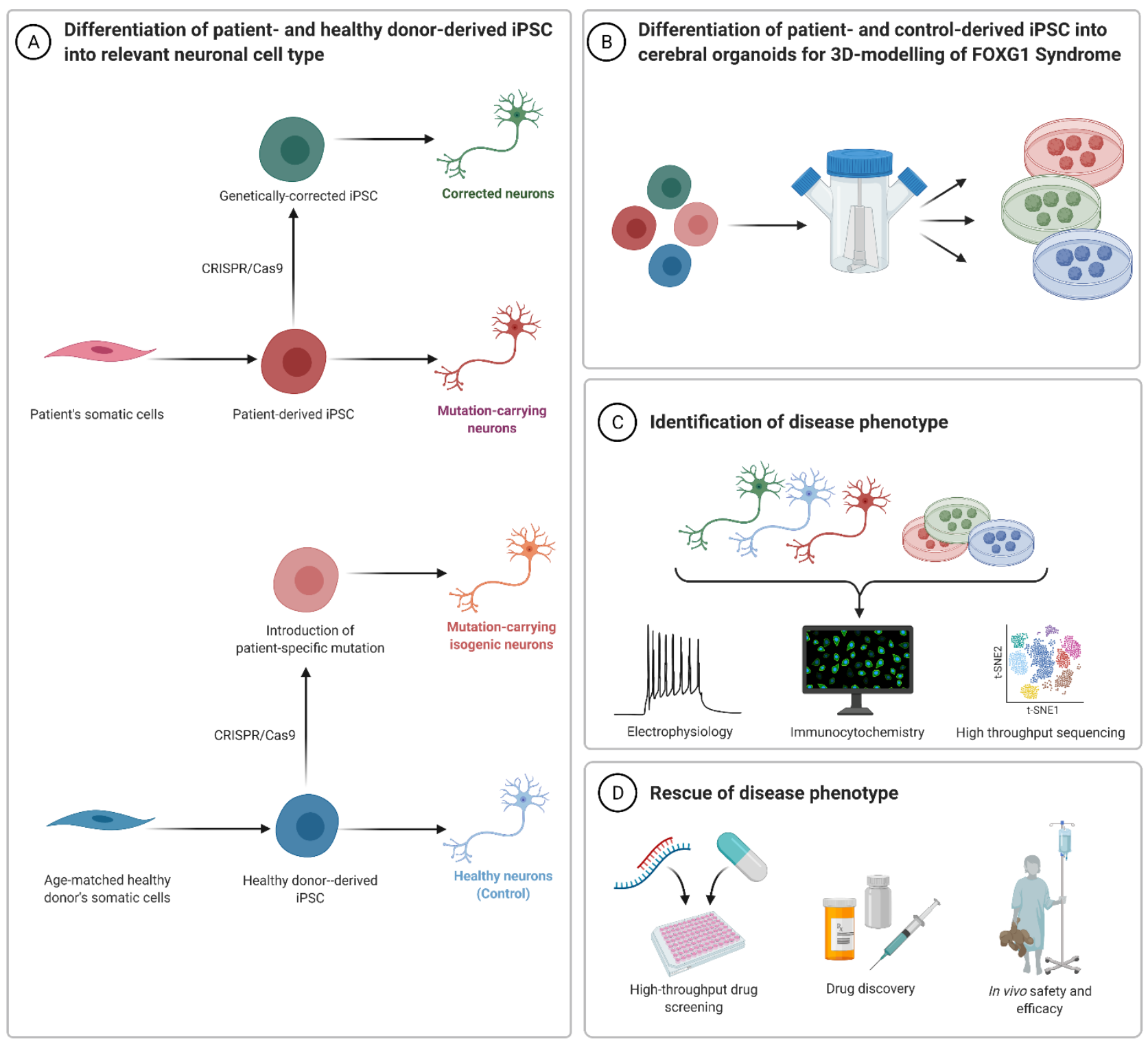

Figure 3: Approaches and potentials of human-based disease modelling for FOXG1 syndrome. (A) Upon genetic screening for FOXG1 syndrome, specific mutations are identified. Patient-derived somatic cells (fibroblast and other cell types) are reprogrammed to a pluripotent state (iPSC). The mutations in these cells are "corrected" using CRISPR/Cas9 approach. In parallel, iPSCs from healthy donors are used to introduce the patient-specific mutations using CRISPR/Cas9 genome editing approaches. iPSCs carrying the disease-related mutation can be differentiated into neural cells type which are affected in the disease. (B) In another approach, iPSCs carrying patient-specific mutations are used to generate cerebral organoids to model neurodevelopment. (C) High throughput sequencing approaches and functional assays are employed on these differentiated neurons and cerebral organoids to recapitulate the syndrome in the human model system. (D) Potential targets and biomarkers obtained from these studies would potentially contribute to drug discoveries and treatment of symptoms in patients. Illustration was created with Biorender.com. 


\section{Acknowledgements:}

IA is supported by funding from the Center for Basics in NeuroModulation (NeuroModul Basics), Medical Faculty, Albert-Ludwigs-University Freiburg, 79104 Freiburg, Germany. This study was also supported by the German Research Foundation 322977937/GRK2344 (TV, IA). The APC was funded by the Baden-Wuerttemberg Ministry of Science, Research and Art and the University of Freiburg in the funding program Open Access Publishing. The figures were created with BioRender.com.

Author contributions: IA and FG wrote the manuscript. TV reviewed and edited the manuscript, and provided conceptual guidance.

Institutional Review Board Statement: Not applicable.

Informed Consent Statement: Not applicable.

Data Availability Statement: Not applicable.

Conflicts of Interest: The authors declare no conflict of interest.

\section{References:}

[1] P.-S. Hou, D. Ó. hAilín, T. Vogel, and C. Hanashima, 'Transcription and Beyond: Delineating FOXG1 Function in Cortical Development and Disorders', Front. Cell. Neurosci., vol. 14, p. 35, Feb. 2020, doi: 10.3389/fncel.2020.00035.

[2] W. Tao and E. Lai, 'Telencephalon-restricted expression of BF-1, a new member of the HNF-3/fork head gene family, in the developing rat brain', Neuron, vol. 8, no. 5, pp. 957-966, May 1992, doi: 10.1016/0896-6273(92)90210-5.

[3] M. Chahrour and H. Y. Zoghbi, 'The Story of Rett Syndrome: From Clinic to Neurobiology', Neuron, vol. 56, no. 3, pp. 422-437, Nov. 2007, doi: 10.1016/j.neuron.2007.10.001.

[4] F. Kortum et al., 'The core FOXG1 syndrome phenotype consists of postnatal microcephaly, severe mental retardation, absent language, dyskinesia, and corpus callosum hypogenesis', Journal of Medical Genetics, vol. 48, no. 6, pp. 396-406, Jun. 2011, doi: 10.1136/jmg.2010.087528.

[5] D. Mitter et al., 'FOXG1 syndrome: genotype-phenotype association in 83 patients with FOXG1 variants', Genet Med, vol. 20, no. 1, pp. 98-108, Jan. 2018, doi:

10.1038/gim.2017.75. 
[6] J. Mariani et al., 'FOXG1-Dependent Dysregulation of GABA/Glutamate Neuron

Differentiation in Autism Spectrum Disorders', Cell, vol. 162, no. 2, pp. 375-390, Jul. 2015, doi: 10.1016/j.cell.2015.06.034.

[7] 'What is FOXG1 syndrome?', FOXG1 Research Foundation. https://foxg1research.org/foxg1 syndrome (accessed Nov. 23, 2021).

[8] C. J. Ellaway et al., ' 14q12 microdeletions excluding FOXG1 give rise to a congenital variant Rett syndrome-like phenotype', Eur J Hum Genet, vol. 21, no. 5, pp. 522-527, May 2013, doi: 10.1038/ejhg.2012.208.

[9] M. Takagi, G. Sasaki, T. Mitsui, M. Honda, Y. Tanaka, and T. Hasegawa, 'A 2.0 Mb microdeletion in proximal chromosome $14 q 12$, involving regulatory elements of FOXG1, with the coding region of FOXG1 being unaffected, results in severe developmental delay, microcephaly, and hypoplasia of the corpus callosum', European Journal of Medical Genetics, vol. 56, no. 9, pp. 526-528, Sep. 2013, doi: 10.1016/j.ejmg.2013.05.012.

[10] N. Brunetti-Pierri et al., 'Duplications of FOXG1 in 14q12 are associated with developmental epilepsy, mental retardation, and severe speech impairment', European journal of human genetics : EJHG, vol. 19, no. 1, pp. 102-107, Jan. 2011, doi: 10.1038/ejhg.2010.142.

[11] N. Vegas et al., 'Delineating FOXG1 syndrome: From congenital microcephaly to hyperkinetic encephalopathy', Neurol Genet, vol. 4, no. 6, p. e281, Dec. 2018, doi: 10.1212/NXG.0000000000000281.

[12] L.-C. Wong, Y.-T. Wu, C.-J. Hsu, W.-C. Weng, W.-C. Tsai, and W.-T. Lee, 'Cognition and Evolution of Movement Disorders of FOXG1-Related Syndrome', Front. Neurol., vol. 10, p. 641, Jun. 2019, doi: 10.3389/fneur.2019.00641.

[13] S. A. Shoichet et al., 'Haploinsufficiency of novel FOXG1B variants in a patient with severe mental retardation, brain malformations and microcephaly', Hum Genet, vol. 117, no. 6, pp. 536-544, Oct. 2005, doi: 10.1007/s00439-005-1310-3.

[14] F. Ariani et al., 'FOXG1 Is Responsible for the Congenital Variant of Rett Syndrome', Am J Hum Genet, vol. 83, no. 1, pp. 89-93, Jul. 2008, doi: 10.1016/j.ajhg.2008.05.015.

[15] C. K. Byun, J. S. Lee, B. C. Lim, K. J. Kim, Y. S. Hwang, and J.-H. Chae, 'FOXG1 Mutation is a Low-Incidence Genetic Cause in Atypical Rett Syndrome', Child Neurology Open, vol. 2, no. 1, p. 2329048X14568151, Mar. 2015, doi: $10.1177 / 2329048 \times 14568151$.

[16] N. Bredenkamp, C. Seoighe, and N. Illing, 'Comparative evolutionary analysis of the FoxG1 transcription factor from diverse vertebrates identifies conserved recognition sites for microRNA regulation', Dev Genes Evol, vol. 217, no. 3, pp. 227-233, Mar. 2007, doi: 10.1007/s00427-006-0128-x.

[17] S. Wiese et al., 'The genes for human brain factor 1 and 2, members of the fork head gene family, are clustered on chromosome 14q', Biochim Biophys Acta, vol. 1262, no. 2-3, pp. 105-112, Jun. 1995, doi: 10.1016/0167-4781(95)00059-p.

[18] N. C. Hettige and C. Ernst, 'FOXG1 Dose in Brain Development', Front. Pediatr., vol. 7, p. 482, Nov. 2019, doi: 10.3389/fped.2019.00482.

[19] T. Kumamoto and C. Hanashima, 'Evolutionary conservation and conversion of Foxg1 function in brain development', Development, Growth \& Differentiation, vol. 59, no. 4, pp. 258-269, 2017, doi: https://doi.org/10.1111/dgd.12367.

[20] J. Li et al., 'Mechanism of forkhead transcription factors binding to a novel palindromic DNA site', Nucleic Acids Research, vol. 49, no. 6, pp. 3573-3583, Apr. 2021, doi: 10.1093/nar/gkab086.

[21] S. Dai, L. Qu, J. Li, and Y. Chen, 'Toward a mechanistic understanding of DNA binding by forkhead transcription factors and its perturbation by pathogenic mutations', Nucleic Acids Research, vol. 49, no. 18, pp. 10235-10249, Oct. 2021, doi: 10.1093/nar/gkab807.

[22] S. Dai et al., 'Structural Basis for DNA Recognition by FOXG1 and the Characterization of Disease-causing FOXG1 Mutations', Journal of Molecular Biology, vol. 432, no. 23, pp. 6146-6156, Nov. 2020, doi: 10.1016/j.jmb.2020.10.007.

[23] C. Caporali, S. Signorini, V. De Giorgis, A. Pichiecchio, O. Zuffardi, and S. Orcesi, 'Early-onset movement disorder as diagnostic marker in genetic syndromes: Three 
cases of FOXG1-related syndrome', European Journal of Paediatric Neurology, vol. 22, no. 2, pp. 336-339, Mar. 2018, doi: 10.1016/j.ejpn.2018.01.007.

[24] L. E. Seltzer et al., 'Epilepsy and outcome in FOXG1-related disorders', Epilepsia, vol. 55, no. 8, pp. 1292-1300, Aug. 2014, doi: 10.1111/epi.12648.

[25] A. Bębenek and I. Ziuzia-Graczyk, 'Fidelity of DNA replication-a matter of proofreading', Curr Genet, vol. 64, no. 5, pp. 985-996, Oct. 2018, doi: 10.1007/s00294018-0820-1.

[26] M. M. Mehrjouy et al., 'Regulatory variants of FOXG1 in the context of its topological domain organisation', Eur J Hum Genet, vol. 26, no. 2, pp. 186-196, Feb. 2018, doi: 10.1038/s41431-017-0011-4.

[27] A. Papandreou et al., 'Delineation of the movement disorders associated with FOXG1 mutations', Neurology, vol. 86, no. 19, pp. 1794-1800, May 2016, doi: 10.1212/WNL.0000000000002585.

[28] S. Xuan, C. A. Baptista, G. Balas, W. Tao, V. C. Soares, and E. Lai, 'Winged helix transcription factor BF-1 is essential for the development of the cerebral hemispheres', Neuron, vol. 14, no. 6, pp. 1141-1152, Jun. 1995, doi: 10.1016/0896-6273(95)90262-7.

[29] C. Hanashima, L. Shen, S. C. Li, and E. Lai, 'Brain Factor-1 Controls the Proliferation and Differentiation of Neocortical Progenitor Cells through Independent Mechanisms', J. Neurosci., vol. 22, no. 15, pp. 6526-6536, Aug. 2002, doi: 10.1523/JNEUROSCI.2215-06526.2002.

[30] C. Hanashima, M. Fernandes, J. M. Hebert, and G. Fishell, 'The Role of Foxg1 and Dorsal Midline Signaling in the Generation of Cajal-Retzius Subtypes', p. 9.

[31] G. Miyoshi and G. Fishell, 'Dynamic FoxG1 Expression Coordinates the Integration of Multipolar Pyramidal Neuron Precursors into the Cortical Plate', Neuron, vol. 74, no. 6, pp. 1045-1058, Jun. 2012, doi: 10.1016/j.neuron.2012.04.025.

[32] C. Hanashima, 'Foxg1 Suppresses Early Cortical Cell Fate', Science, vol. 303, no. 5654, pp. 56-59, Jan. 2004, doi: 10.1126/science.1090674.

[33] T. Kumamoto et al., 'Foxg1 Coordinates the Switch from Non-Radially to Radially Migrating Glutamatergic Subtypes in the Neocortex through Spatiotemporal Repression', Cell Rep, vol. 3, no. 3, pp. 931-945, Mar. 2013, doi: 10.1016/j.celrep.2013.02.023.

[34] P.-S. Hou, G. Miyoshi, and C. Hanashima, 'Sensory cortex wiring requires preselection of short- and long-range projection neurons through an Egr-Foxg1-COUP-TFI network', Nature Communications, vol. 10, no. 1, p. 3581, Aug. 2019, doi: 10.1038/s41467-01911043-w.

[35] F. Cargnin, J.-S. Kwon, S. Katzman, B. Chen, J. W. Lee, and S.-K. Lee, 'FOXG1 Orchestrates Neocortical Organization and Cortico-Cortical Connections', Neuron, vol. 100, no. 5, pp. 1083-1096.e5, Dec. 2018, doi: 10.1016/j.neuron.2018.10.016.

[36] J. M. Hébert and S. K. McConnell, 'Targeting of cre to the Foxg1 (BF-1) Locus Mediates loxP Recombination in the Telencephalon and Other Developing Head Structures', Developmental Biology, vol. 222, no. 2, pp. 296-306, Jun. 2000, doi: 10.1006/dbio.2000.9732.

[37] L. Shen, H.-S. Nam, P. Song, H. Moore, and S. A. Anderson, 'FoxG1 haploinsufficiency results in impaired neurogenesis in the postnatal hippocampus and contextual memory deficits', p. 16, 2006.

[38] K. L. Eagleson et al., 'Disruption of Foxg1 expression by knock-in of Cre recombinase: Effects on the development of the mouse telencephalon', Neuroscience, vol. 148, no. 2, pp. 385-399, Aug. 2007, doi: 10.1016/j.neuroscience.2007.06.012.

[39] J. A. Siegenthaler, B. A. Tremper-Wells, and M. W. Miller, 'Foxg1 Haploinsufficiency Reduces the Population of Cortical Intermediate Progenitor Cells: Effect of Increased p21 Expression', Cerebral Cortex, vol. 18, no. 8, pp. 1865-1875, Aug. 2008, doi: 10.1093/cercor/bhm209.

[40] C. Dou et al., 'BF-1 interferes with transforming growth factor beta signaling by associating with Smad partners', Mol Cell Biol, vol. 20, no. 17, pp. 6201-6211, Sep. 2000, doi: 10.1128/MCB.20.17.6201-6211.2000.

[41] J. Seoane, H.-V. Le, L. Shen, S. A. Anderson, and J. Massagué, 'Integration of Smad and Forkhead Pathways in the Control of Neuroepithelial and Glioblastoma Cell 
Proliferation', Cell, vol. 117, no. 2, pp. 211-223, Apr. 2004, doi: 10.1016/S00928674(04)00298-3.

[42] R. Vezzali, S. C. Weise, N. Hellbach, S. Heidrich, and T. Vogel, 'The FOXG1/FOXO/SMAD network balances proliferation and differentiation of cortical progenitors and activates Kcnh3 expression in mature neurons', p. 20, 2016.

[43] S. K. McConnell, 'Constructing the cerebral cortex: neurogenesis and fate determination', Neuron, vol. 15, no. 4, pp. 761-768, Oct. 1995, doi: 10.1016/08966273(95)90168-x.

[44] G. Agirman, L. Broix, and L. Nguyen, 'Cerebral cortex development: an outside-in perspective', FEBS Lett, vol. 591, no. 24, pp. 3978-3992, Dec. 2017, doi: 10.1002/1873-3468.12924.

[45] C. R. Cadwell, A. Bhaduri, M. A. Mostajo-Radji, M. G. Keefe, and T. J. Nowakowski, 'Development and Arealization of the Cerebral Cortex', Neuron, vol. 103, no. 6, pp. 980-1004, Sep. 2019, doi: 10.1016/j.neuron.2019.07.009.

[46] R. M. Fame, J. L. MacDonald, and J. D. Macklis, 'Development, specification, and diversity of callosal projection neurons', Trends Neurosci, vol. 34, no. 1, pp. 41-50, Jan. 2011, doi: 10.1016/j.tins.2010.10.002.

[47] G. Testa et al., 'A triheptanoin-supplemented diet rescues hippocampal hyperexcitability and seizure susceptibility in FoxG1+/- mice', Neuropharmacology, vol. 148, pp. 305-310, Apr. 2019, doi: 10.1016/j.neuropharm.2019.01.005.

[48] G. Testa et al., 'Cortical Seizures in FoxG1+/- Mice are Accompanied by Akt/S6 Overactivation, Excitation/Inhibition Imbalance and Impaired Synaptic Transmission', IJMS, vol. 20, no. 17, p. 4127, Aug. 2019, doi: 10.3390/ijms20174127.

[49] L. Pancrazi et al., 'Foxg1 localizes to mitochondria and coordinates cell differentiation and bioenergetics', Proc Natl Acad Sci U S A, vol. 112, no. 45, pp. 13910-13915, Nov. 2015, doi: 10.1073/pnas.1515190112.

[50] G. M. Liebhaber, E. Riemann, and F. A. Matthias Baumeister, 'Ketogenic Diet in Rett Syndrome', J Child Neurol, vol. 18, no. 1, pp. 74-75, Jan. 2003, doi: $10.1177 / 08830738030180011801$.

[51] J. G. Mantis, N. A. Centeno, M. T. Todorova, R. McGowan, and T. N. Seyfried, 'Management of multifactorial idiopathic epilepsy in EL mice with caloric restriction and the ketogenic diet: role of glucose and ketone bodies', Nutr Metab (Lond), vol. 1, no. 1, p. 11, Oct. 2004, doi: 10.1186/1743-7075-1-11.

[52] M. J. Park et al., 'Anaplerotic Triheptanoin Diet Enhances Mitochondrial Substrate Use to Remodel the Metabolome and Improve Lifespan, Motor Function, and Sociability in MeCP2-Null Mice', PLOS ONE, vol. 9, no. 10, p. e109527, Oct. 2014, doi: 10.1371/journal.pone.0109527.

[53] T. Patriarchi et al., 'Imbalance of excitatory/inhibitory synaptic protein expression in iPSC-derived neurons from FOXG1(+/-) patients and in foxg1(+/-) mice.', European journal of human genetics : EJHG, vol. 24, no. 6, pp. 871-880, Jun. 2016, doi: 10.1038/ejhg.2015.216.

[54] S. C. Weise et al., 'FOXG1 Regulates PRKAR2B Transcriptionally and Posttranscriptionally via miR200 in the Adult Hippocampus', Mol Neurobiol, vol. 56, no. 7, pp. 5188-5201, Jul. 2019, doi: 10.1007/s12035-018-1444-7.

[55] A. R. Gomes, T. G. Fernandes, J. M. S. Cabral, and M. M. Diogo, 'Modeling Rett Syndrome with Human Pluripotent Stem Cells: Mechanistic Outcomes and Future Clinical Perspectives.', International journal of molecular sciences, vol. 22, no. 7, Apr. 2021, doi: 10.3390/ijms22073751.

[56] M. C. N. Marchetto et al., 'A model for neural development and treatment of Rett syndrome using human induced pluripotent stem cells.', Cell, vol. 143, no. 4, pp. 527539, Nov. 2010, doi: 10.1016/j.cell.2010.10.016.

[57] G. Livide et al., 'GluD1 is a common altered player in neuronal differentiation from both MECP2-mutated and CDKL5-mutated iPS cells.', European journal of human genetics : EJHG, vol. 23, no. 2, pp. 195-201, Feb. 2015, doi: 10.1038/ejhg.2014.81.

[58] S. Croci et al., 'AAV-mediated FOXG1 gene editing in human Rett primary cells.', European journal of human genetics : EJHG, vol. 28, no. 10, pp. 1446-1458, Oct. 2020, doi: 10.1038/s41431-020-0652-6. 
[59] A. J. Waite, D. Millar, and A. Clarke, 'The generation of an induced pluripotent stem cell line (DCGi001-A) from an individual with FOXG1 syndrome carrying the c.460dupG (p.Glu154fs) variation in the FOXG1 gene', Stem Cell Research, vol. 49, p. 102018, Dec. 2020, doi: 10.1016/j.scr.2020.102018.

[60] K. Sampieri et al., 'Italian Rett database and biobank.', Human mutation, vol. 28, no. 4, pp. 329-335, Apr. 2007, doi: 10.1002/humu.20453.

[61] N. Sun, X. Meng, Y. Liu, D. Song, C. Jiang, and J. Cai, 'Applications of brain organoids in neurodevelopment and neurological diseases', Journal of Biomedical Science, vol. 28, no. 1, p. 30, 2021, doi: 10.1186/s12929-021-00728-4.

[62] M. A. Lancaster and J. A. Knoblich, 'Generation of cerebral organoids from human pluripotent stem cells.', Nature protocols, vol. 9, no. 10, pp. 2329-2340, Oct. 2014, doi: 10.1038/nprot.2014.158.

[63] W. Zhu et al., 'Precisely controlling endogenous protein dosage in hPSCs and derivatives to model FOXG1 syndrome', Nat Commun, vol. 10, no. 1, p. 928, Dec. 2019, doi: 10.1038/s41467-019-08841-7.

[64] R. A. Samarasinghe et al., 'Identification of neural oscillations and epileptiform changes in human brain organoids', Nature Neuroscience, vol. 24, no. 10, pp. 1488-1500, 2021, doi: 10.1038/s41593-021-00906-5.

[65] A. R. Gomes et al., 'Modeling Rett Syndrome With Human Patient-Specific Forebrain Organoids.', Frontiers in cell and developmental biology, vol. 8, p. 610427, 2020, doi: 10.3389/fcell.2020.610427.

[66] D. Sun et al., 'A functional genetic toolbox for human tissue-derived organoids', Elife, vol. 10, p. e67886, Oct. 2021, doi: 10.7554/eLife.67886.

[67] M. T. Pham et al., 'Generation of human vascularized brain organoids', Neuroreport, vol. 29, no. 7, pp. 588-593, May 2018, doi: 10.1097/WNR.0000000000001014.

[68] Y. Shi et al., 'Vascularized human cortical organoids (vOrganoids) model cortical development in vivo', PLOS Biology, vol. 18, no. 5, p. e3000705, May 2020, doi: 10.1371/journal.pbio.3000705.

[69] Y. Ahn et al., 'Human Blood Vessel Organoids Penetrate Human Cerebral Organoids and Form a Vessel-Like System', Cells, vol. 10, no. 8, p. 2036, Aug. 2021, doi: $10.3390 /$ cells 10082036.

[70] S. Bergmann et al., 'Blood-brain-barrier organoids for investigating the permeability of CNS therapeutics', Nat Protoc, vol. 13, no. 12, pp. 2827-2843, Dec. 2018, doi: 10.1038/s41596-018-0066-x.

[71] J. A. Bagley, D. Reumann, S. Bian, J. Lévi-Strauss, and J. A. Knoblich, 'Fused cerebral organoids model interactions between brain regions', Nat Methods, vol. 14, no. 7, pp. 743-751, Jul. 2017, doi: 10.1038/nmeth.4304.

[72] E. Gabriel et al., 'Human brain organoids assemble functionally integrated bilateral optic vesicles', Cell Stem Cell, vol. 28, no. 10, pp. 1740-1757.e8, Oct. 2021, doi: 10.1016/j.stem.2021.07.010.

[73] J. D. Pereira et al., 'Human sensorimotor organoids derived from healthy and amyotrophic lateral sclerosis stem cells form neuromuscular junctions', Nat Commun, vol. 12, no. 1, p. 4744, Aug. 2021, doi: 10.1038/s41467-021-24776-4.

[74] J.-M. Faustino Martins et al., 'Self-Organizing 3D Human Trunk Neuromuscular Organoids', Cell Stem Cell, vol. 26, no. 2, pp. 172-186.e6, Feb. 2020, doi: 10.1016/j.stem.2019.12.007.

[75] S. Lenin et al., 'A Drug Screening Pipeline Using 2D and 3D Patient-Derived In Vitro Models for Pre-Clinical Analysis of Therapy Response in Glioblastoma', Int J Mol Sci, vol. 22, no. 9, p. 4322, Apr. 2021, doi: 10.3390/ijms22094322.

[76] Y. Li, P. Tang, S. Cai, J. Peng, and G. Hua, 'Organoid based personalized medicine: from bench to bedside', Cell Regeneration, vol. 9, Dec. 2020, doi: 10.1186/s13619-02000059-z.

[77] J. Azar et al., 'The Use of Stem Cell-Derived Organoids in Disease Modeling: An Update', International Journal of Molecular Sciences, vol. 22, no. 14, p. 7667, Jan. 2021, doi: 10.3390/ijms22147667.

[78] A. Lavazza and M. Massimini, 'Cerebral organoids: ethical issues and consciousness assessment', J Med Ethics, vol. 44, no. 9, pp. 606-610, Sep. 2018, doi: 10.1136/medethics-2017-104555. 
